\title{
Hybrid Spline-Based Elastic Image Registration Using Analytic Solutions of the Navier Equation
}

\author{
Stefan Wörz and Karl Rohr \\ University of Heidelberg, IPMB, and DKFZ Heidelberg, \\ Dept. Bioinformatics and Functional Genomics, Biomedical Computer Vision Group \\ Im Neuenheimer Feld 364, 69120 Heidelberg, Germany \\ Email: s.woerz@dkfz.de
}

\begin{abstract}
We introduce a new hybrid approach for spline-based elastic image registration using both point landmarks and intensity information. As underlying deformation model we use Gaussian elastic body splines (GEBS), which are solutions of the Navier equation of linear elasticity under Gaussian forces. We also incorporate landmark localization uncertainties represented by weight matrices to cope with anisotropic errors. The hybrid registration approach is formulated as an energy-minimizing functional that incorporates landmark and intensity information as well as a regularization based on GEBS. Since the approach is based on a physical deformation model, cross-effects in elastic deformations can be taken into account. We demonstrate the applicability of our scheme based on MR images of the brain. It turns out that the new scheme achieves more accurate results compared to a pure landmark-based as well as a pure intensity-based scheme.
\end{abstract}

\section{Introduction}

The registration of biomedical images is an important task, however, it is difficult and challenging. One reason is that in many applications it is still not quite clear which type of image information is optimal for matching. Another reason is that the spectrum of possible geometric differences is relatively large. Previous work on biomedical image registration can be characterized based on the nature of the transformation (e.g., rigid, nonrigid) as well as on the used image information (e.g., landmark-based, intensity-based). While rigid registration schemes are computationally efficient, they do not allow to cope with local differences between corresponding image data. Therefore, nonrigid (elastic) registration schemes are required (for a survey see [1]). Regarding the used image information, approaches are often based on either landmarks or intensity information. Main advantages of landmark-based approaches are computational efficiency, the fact that they can cope with large geometric differences, and the easy and intuitive incorporation of user-interaction. In contrast, main advantages of intensity-based approaches are that more image information is taken into account and that no segmentation is necessary (higher level of automation). 
Elastic registration schemes are generally based on an energy functional or the related partial differential equation. One possibility is to numerically compute solutions using finite differences or the finite element method, which, however, is computationally expensive. For numeric schemes to improve the efficiency, see, for example, [2]. Alternatively, spline-based approaches can be used for elastic registration, which are often based on a nonuniform grid of control points (landmarks). Examples of such schemes are based on thin-plate splines (TPS, e.g., [3]), elastic body splines (EBS, [4]), and Gaussian EBS (GEBS, e.g., [5]-[8]). TPS are based on the bending energy of a thin plate, which represents a relatively coarse deformation model. In comparison, EBS and GEBS are derived from the Navier equation (partial differential equation), which describes the deformation of elastic tissues (bodies) under certain forces. GEBS in comparison to EBS have the advantage that more realistic image forces are used (Gaussian instead of polynomial forces).

Over the past few years, approaches that combine landmark-based and intensity-based methods have gained increased interest since advantages of both types of methods can be combined. However, so far only few spline-based registration approaches exist that use both landmarks and intensity information (e.g., $[9,10,6,7])$. Typically, the intensity information is only used to determine optimal positions of the control points (e.g., $[6,7]$ ) or to establish landmark correspondences, i.e. the landmarks and intensity information are not directly combined. In addition, often a physical deformation model is not used (e.g., $[9,10])$. Furthermore, in landmark-based approaches generally an interpolation scheme is applied that forces corresponding landmarks to exactly match each other (e.g., $[4,5,6])$. The underlying assumption is that the landmark positions are known exactly. In real applications, however, landmark extraction is always prone to error. Therefore, to take these localization uncertainties into account, approximation schemes have been proposed, e.g., for TPS [3] and GEBS [8]. Note, however, that in these approaches only landmarks have been used but not intensity information.

In contrast to previous spline-based approaches, the central idea of our new approach is to directly combine the landmark and intensity information in a single energy functional as well as to include a regularization based on GEBS. In addition, we incorporate landmark localization uncertainties to cope with anisotropic errors. Since GEBS include a material parameter (Poisson ratio) that defines the ratio between transverse contraction and longitudinal dilation of an elastic material, cross-effects can be taken into account (which is not the case for, e.g., TPS). Moreover, since GEBS incorporate Gaussian forces we have a free parameter (the standard deviation) to control the locality of the transformation, and, therefore, GEBS are well suited for the registration of local differences.

\section{Hybrid Gaussian elastic body splines (GEBS)}

We have developed a new hybrid approach for spline-based elastic image registration using both landmarks and intensity information. As underlying deformation 
model we use Gaussian elastic body splines (GEBS), which are derived from the Navier equation (for details see, e.g., $[5,8]$ ). To compute the deformation field $\mathbf{u}$ for registering the source image $g_{1}$ with the target image $g_{2}$ based on landmark and intensity information, we introduce an energy-minimizing functional $J_{\text {Hybrid }}(\mathbf{u})$, which consists of four terms:

$$
J_{\text {Hybrid }}=J_{\text {Data }, I}\left(g_{1}, g_{2}, \mathbf{u}^{I}\right)+\lambda_{I}\left\|\mathbf{u}^{I}-\mathbf{u}\right\|^{2}+\lambda_{L}\left\|\mathbf{u}^{L}-\mathbf{u}\right\|^{2}+\lambda_{E l} J_{E l}(\mathbf{u})
$$

Besides the searched deformation field $\mathbf{u}$, the functional comprises two deformation fields $\mathbf{u}^{I}$ and $\mathbf{u}^{L}$, which are computed based on the intensity and landmark information, respectively $\left(\lambda_{I}, \lambda_{L}\right.$, and $\lambda_{E l}$ are scalar weights).

Concerning the intensity information, the first term of (1) represents an intensity similarity measure between the deformed source image and the target image. Here, we use the sum-of-squared intensity differences as similarity measure. The second term couples the intensity-based deformation field $\mathbf{u}^{I}$ with $\mathbf{u}$ using the Euclidean distance between both deformation fields.

Regarding the landmark information, the deformation field $\mathbf{u}^{L}$ is computed based on the landmark correspondences using GEBS. To incorporate localization uncertainties of landmarks, we employ the approximation scheme proposed in [8]. With this scheme the landmarks are individually weighted according to their localization uncertainties, which allows to control the influence of the landmarks on the registration result. The localization uncertainties are characterized by weight matrices, i.e. anisotropic landmark errors are taken into account. The third term of (1) couples the landmark-based deformation field $\mathbf{u}^{L}$ with $\mathbf{u}$.

Finally, the fourth term represents the regularization of the deformation field u. In our case, $J_{E l}$ is based on the matrix-valued basis function of GEBS. By minimizing the functional $J_{\mathrm{Hybrid}}$, the resulting deformation field $\mathbf{u}$ is, on the one hand, similar to the deformation field obtained from the landmark correspondences, and, on the other hand, the intensities of the deformed source image are similar to those of the target image. In addition, the regularization using GEBS constraints the deformation field to physically plausible deformations.

An efficient way of minimizing $J_{\text {Hybrid }}$ is to minimize it alternatingly w.r.t. $\mathbf{u}^{I}$ and $\mathbf{u}$. For the minimization w.r.t. $\mathbf{u}^{I}$, the following functional is relevant

$$
J_{\text {Data }, I}\left(g_{1}, g_{2}, \mathbf{u}^{I}\right)+\lambda_{I}\left\|\mathbf{u}^{I}-\mathbf{u}\right\|^{2}
$$

This functional has the advantage that it can be stated independently for each voxel, and that for each voxel only sums of squared differences are used. Therefore, (2) can be efficiently minimized using the method of Levenberg/Marquardt. For the minimization w.r.t. $\mathbf{u}$, the following functional has to be considered

$$
\lambda_{I}\left\|\mathbf{u}^{I}-\mathbf{u}\right\|^{2}+\lambda_{L}\left\|\mathbf{u}^{L}-\mathbf{u}\right\|^{2}+\lambda_{E l} J_{E l}(\mathbf{u})
$$

Interestingly, for minimizing (3) an explicit solution can be stated $\mathbf{u}(\mathbf{x})=\mathbf{G}(\mathbf{x}) *$ $\left[\mathbf{u}^{I}(\mathbf{x})-\mathbf{u}^{L}(\mathbf{x})\right]+\mathbf{u}^{L}(\mathbf{x})$, where " $*$ " denotes the convolution of a matrix-valued function with a vector field and $\mathbf{G}$ is the matrix-valued GEBS basis function. 
Fig. 1. Registration of 2D MR brain images: Pre- (top left) and postsurgical image (bottom left) as well as the (inverse) deformation fields (top) and registered source images (bottom) using a pure landmark-based approach (middle left), a pure intensitybased approach (middle right), and using the new hybrid approach (right)
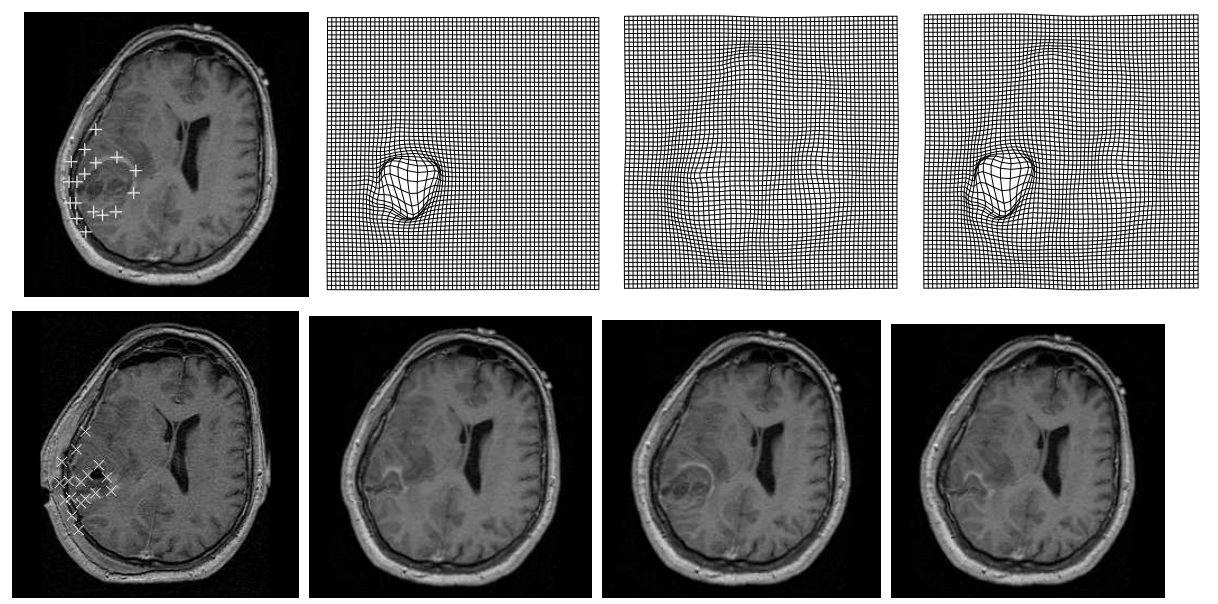

\section{$3 \quad$ Experimental results}

We have applied the new hybrid registration approach to register 2D MR images of the human head. In this application the task is to register pre- and postsurgical MR images of the human brain. Fig. 1 shows 2D MR images of a patient before (source image, top left) and after (target image, bottom left) the resection of a tumor. 17 landmarks have been manually placed along the contours of the tumor and the resection area (indicated by crosses). Fig. 1 shows the (inverse) deformation fields (top) and registered source images (bottom) using a pure landmark-based approach (middle left), a pure intensity-based approach (middle right), and using the new hybrid approach based on GEBS (right). It turned out that using only landmark information (middle left) the vicinity of the tumor and resection area are well registered whereas regions without landmarks are not deformed. As a consequence, the mean intensity error improved by only $4.6 \%$ w.r.t. the unregistered case. In contrast, using only intensity information (middle right) yields deformations in different parts of the head with an improvement of the mean intensity error by $12.8 \%$. However, the tumor has not been registered. Using the new hybrid approach the registration result is significantly improved in comparison to the previous two approaches since the tumor and resection area are well registered and, in addition, other parts of the head (see Fig. 1, right). Here, the mean intensity error for the whole image improved by $13.0 \%$. 


\section{Conclusion}

The presented hybrid elastic approach combines landmarks and intensity information as well as a regularization based on Gaussian elastic body splines (GEBS), which are analytic solutions of the Navier equation. In comparison to existing spline-based approaches, the new scheme combines a number of advantages. A main advantage is that the hybrid approach directly combines landmarks and intensity information, and hence exploits advantages from both landmark-based and intensity-based approaches. In contrast, existing splinebased approaches often use intensity information only to localize control points or to establish landmark correspondences. Moreover, the new registration approach takes into account anisotropic localization uncertainties of the landmark positions. We have demonstrated the applicability of our new registration approach based on MR brain images. From the experiments it turned out that the hybrid approach achieves more accurate registration results in comparison to a pure landmark-based approach and a pure intensity-based scheme.

\section{Acknowledgment}

This work has been funded by the Deutsche Forschungsgemeinschaft (DFG) within the project ELASTIR (RO 2471/2). The original images and the tumor outlines have kindly been provided by OA Dr. med. U. Spetzger and Prof. Dr. J.M. Gilsbach, Neurosurgical Clinic, University Hospital Aachen of the RWTH.

\section{References}

1. Zitova B, Flusser J. Image registration methods: A survey. Image and Vision Computing 2003;24:977-1000.

2. Modersitzki J, Fischer B. Optimal image registration with a guaranteed one-to-one point match. Procs BVM 2003; 1-5.

3. Rohr K, Stiehl HS, Sprengel R, et al. Landmark-based elastic registration using approximating thin-plate splines. IEEE Trans Med Imaging 2001;20(6):526-534.

4. Davis MH, Khotanzad A, Flaming DP, et al. A physics-based coordinate transformation for 3D image matching. IEEE Trans Med Imaging 1997;16(3):317-328.

5. Kohlrausch J, Rohr K, Stiehl HS. A new class of elastic body splines for nonrigid registration of medical images. J Mathematical Imaging and Vision 2005;23(3):253280.

6. Pekar V, Gladilin E, Rohr K. An adaptive irregular grid approach for 3-D deformable image registration. Phys Med Biol 2006;51:361-377.

7. Franz A, Carlson IC, Renisch S. An adaptive irregular grid approach using SIFT features for elastic medical image registration. Procs BVM 2006; 201-205.

8. Wörz S, Rohr K. Physics-based elastic image registration using splines and including landmark localization uncertainties. LNCS 2006;4191:678-685.

9. Cachier P, Mangin JF, Pennec X, et al. Multisubject non-rigid registration of brain MRI using intensity and geometric features. LNCS 2001;2208:734-742.

10. Rohr K, Cathier P, Wörz S. Elastic registration of electrophoresis images using intensity information and point landmarks. Pattern Recognition 2004;37(5):10351048 . 